ARTICLE

\title{
Cryo-EM structure of trimeric Mycobacterium smegmatis succinate dehydrogenase with a membrane-anchor SdhF
}

\author{
Hongri Gong (1) 1,10凶 , Yan Gao 2,10, Xiaoting Zhou ${ }^{3}$, Yu Xiao ${ }^{3}$, Weiwei Wang 3 , Yanting Tang', Shan Zhou', \\ Yuying Zhang ${ }^{1}$, Wenxin $\mathrm{Ji}^{4}$, Lu Yu (D) ${ }^{5}$, Changlin Tian ${ }^{5,6}$, Sin Man Lam7, Guanghou Shui (D) ${ }^{7}$, Luke W. Guddat (i) ${ }^{8}$, \\ Luet-Lok Wong (1) ${ }^{9}$, Quan Wang (10 ${ }^{3,4 凶}$ \& Zihe Rao (1) $1,2,3,4 凶$
}

Diheme-containing succinate:menaquinone oxidoreductases (Sdh) are widespread in Grampositive bacteria but little is known about the catalytic mechanisms they employ for succinate oxidation by menaquinone. Here, we present the $2.8 \AA$ cryo-electron microscopy structure of a Mycobacterium smegmatis Sdh, which forms a trimer. We identified the membrane-anchored $\mathrm{SdhF}$ as a subunit of the complex. The $3 \mathrm{kDa} \mathrm{SdhF}$ forms a single transmembrane helix and this helix plays a role in blocking the canonically proximal quinone-binding site. We also identified two distal quinone-binding sites with bound quinones. One distal binding site is formed by neighboring subunits of the complex. Our structure further reveals the electron/ proton transfer pathway for succinate oxidation by menaquinone. Moreover, this study provides further structural insights into the physiological significance of a trimeric respiratory complex II. The structure of the menaquinone binding site could provide a framework for the development of Sdh-selective anti-mycobacterial drugs.

\footnotetext{
${ }^{1}$ State Key Laboratory of Medicinal Chemical Biology, Frontiers Science Center for Cell Responses, College of Life Sciences, Nankai University, 300353 Tianjin, China. ${ }^{2}$ Laboratory of Structural Biology, Tsinghua University, 100084 Beijing, China. ${ }^{3}$ Shanghai Institute for Advanced Immunochemical Studies and School of Life Science and Technology, ShanghaiTech University, 201210 Shanghai, China. ${ }^{4}$ National Laboratory of Biomacromolecules,omolecules, Institute of Biophysics, CAS, 100101 Beijing, China. ${ }^{5}$ High Magnetic Field Laboratory, Chinese Academy of Sciences, 230031 Hefei, China. ${ }^{6}$ Hefei National Laboratory of Physical Sciences at Microscale and School of Life Sciences, University of Science and Technology of China, 230027 Hefei, China. ${ }^{7}$ State Key Laboratory of Molecular Developmental Biology, Institute of Genetics and Developmental Biology, CAS, 100101 Beijing, China. ${ }^{8}$ School of Chemistry and Molecular Biosciences, The University of Queensland, Brisbane 4072 QLD, Australia. ${ }^{9}$ Department of Chemistry, Inorganic Chemistry Laboratory, University of Oxford, South Parks Road, Oxford OX1 3QR, UK. ${ }^{10}$ These authors contributed equally: Hongri Gong, Yan Gao. ${ }^{凶}$ email: gonghr@nankai.edu.cn; wangq@ibp.ac.cn; raozh@tsinghua.edu.cn
} 
C ellular respiration is an essential feature in the metabolism of many living cells. During respiration, electrons from the compounds in food are transferred to terminal electron acceptors through the electron transport chain (ETC), which couples the translocation of protons across a membrane (either cellular or mitochondrial). This creates a transmembrane proton gradient for a variety of processes, such as the synthesis of ATP (adenosine triphosphate). Electrons enter the ETC through either respiratory complex I or complex $\mathrm{II}^{1,2}$.

Complex II is both a functional member of the oxidative phosphorylation pathway and the Krebs cycle, thus linking these two energy-harvesting processes. Specifically, its roles are to catalyze the reversible oxidoreduction of succinate and fumarate in a soluble domain, coupled to the reversible oxidoreduction of quinone and quinol in a membrane-spanning domain $^{3}$. Members of the complex II superfamily are identified as succinate:quinone oxidoreductases (SQRs; or succinate dehydrogenases, SDHs) or quinol:fumarate oxidoreductases (QFRs; or fumarate reductases, FRDs) according to the preferred direction of reaction ${ }^{3,4}$. No matter the direction, SQRs and QFRs share a common architecture with a large soluble domain and a smaller integral-membrane domain. They are comprised of a soluble flavin-containing subunit, an iron-sulfur cluster-containing subunit, either one or two transmembrane subunits and either zero, one, or two $b$-type heme groups. As a result, the membrane-spanning region has been regarded as the distinct evolutionary origin. Thus, the complex II superfamily has been further divided into type A-F subfamilies according to the number of transmembrane polypeptides and $b$-type hemes within the membrane-spanning region ${ }^{5}$. Intriguingly, the $\mathrm{X}$-ray crystal structures have demonstrated that the oligomerization state of the complex II protein differs both across the superfamily and within subfamilies with monomeric $c^{6,7}$, dimeric ${ }^{8-10}$, and trimeric ${ }^{11}$ states all observed.

The diheme-containing family of complex II, namely type $A$ and $B$ subfamilies, found in a wide range of bacteria, is of particular interest, because its members support transmembrane electron transfer ${ }^{12}$. This process may or may not be coupled to transmembrane proton transfer, depending on the species and the direction of the reaction catalyzed in vivo ${ }^{13}$. The protoncoupled electron transfer events of specific diheme QFRs have already been studied based on the X-ray crystal structures from Wolinella succinogenes $^{9,14,15}$ and Desulfovibrio gigas ${ }^{10}$. In spite of major advances in understanding the electron/proton transfer in diheme-containing QFRs, little is known about the molecular mechanism in the diheme-containing SQRs. Diheme-containing SQRs generally perform menaquinone-linked succinate oxidation $^{12}$, a thermodynamically unfavorable reaction ${ }^{16}$. It has been proposed that the endergonic reduction of menaquinone by succinate is driven by the electrochemical proton potential ${ }^{17-19}$. The diheme-containing SQRs, performing menaquinonedependent succinate oxidation, that have been isolated from Thermus thermophiles ${ }^{20,21}$, Bacillus licheniformis ${ }^{18}$, and Corynebacterium glutamicum ${ }^{22}$ exist in trimeric forms and exhibit positive cooperativity ${ }^{20,21}$. In addition, the dihemecontaining SQR also plays pivotal cellular and energetic roles in mycobacteria, including Mycobacterium smegmatis ${ }^{23}$ and Mycobacterium tuberculosis ${ }^{24,25}$. Therefore, structural data for the diheme-containing SQRs can explain how electron-proton transfer is coupled in this thermodynamically unfavorable reaction. In the present study, we obtained a $2.8 \AA$ cryo-electron microscopy (cryo-EM) structure of a trimeric form of an Sdh2 protein from $M$. smegmatis, which will help us to understand the mechanism of this catalytic process in the dihemecontaining SQRs.

\section{Results and discussion}

Purification and characterization of M. smegmatis Sdh2. Most mycobacterial species including $M$. smegmatis harbor two putative genes encoding different SDHs designated Sdh1 and Sdh2 ${ }^{26}$. Sdh2 has been reported to contain two $b$-type heme groups embedded in the membrane-bound domain ${ }^{23}$. To isolate the Sdh2 in a functional form, protein purification was performed according to the method described previously ${ }^{27}$. Nickel-nitrilotriacetic acid (Ni-NTA) affinity chromatography and gel filtration were used to obtain a purified supramolecular assembly (Supplementary Fig. 1a). All the expected components (subunits A, B, C, and D) of Sdh2 as well as an unknown component (subunit $\mathrm{F}$, described below) were detected by sodium dodecyl sulfate-polyacrylamide gel electrophoresis (SDSPAGE) (Supplementary Fig. 1b) and mass spectrometry (MS) (Supplementary Table 1). A single band on blue native PAGE (BNPAGE) indicated that SDH is present (Supplementary Fig. 1c). Electronic absorption spectra and electron paramagnetic resonance (EPR) spectra showed signals that correspond to the prosthetic groups ([2Fe-2S], [4Fe-4S], [3Fe-4S], and two $b$-type heme groups), all required for electron transfer (Supplementary Fig. 1d, e). Sdh2 oxidized succinate with an apparent catalytic constant $\left(k_{\text {cat }}\right)$ of $3.16 \pm 0.07 \mathrm{~s}^{-1}$ and a Michaelis constant $\left(K_{\mathrm{m}}\right)$ of $65.82 \pm 6.46 \mu \mathrm{M}$ (Supplementary Fig. 6). The data confirm that the purified Sdh2 is a functioning complex that couples succinate oxidation to menadione reduction.

Structural overview. The structure of Sdh2 was determined by cryo-EM to an overall resolution of $2.8 \AA$ (Supplementary Figs. 2 and 3 and Supplementary Table 2). The dimensions of the Sdh2 trimer are $110 \AA$ in width and $105 \AA$ in height, adopting a mushroom-like shape (Fig. 1a). The form of trimeric assembly is similar to that observed in the Escherichia coli SQR ${ }^{11}$. In this structure, we observe extensive contacts between neighboring protomers of the trimer on both the cytoplasmic and membranespanning regions, suggesting that they are important for the assembly and stability of the trimer. Within the trimer, each of the three assemblies contains four canonical proteins: an FAD (flavin adenine dinucleotide)-binding protein (SdhA), an ironsulfur protein $(\mathrm{SdhB})$, and two membrane-anchored proteins (SdhC and SdhD), each with three transmembrane helices (Figs. $1 \mathrm{~b}$ and $2 \mathrm{a}, \mathrm{b}$ ). Its subunit composition is similar to complex II from porcine and $E$. coli SQRs ${ }^{6,11}$. The SdhA subunit also can be divided into four domains ${ }^{6,8}$. Its capping domain is significantly disordered in the cryo-EM map. In other previously reported complexes, substrate ${ }^{28}$ and ligand ${ }^{29}$ binding has been shown to result in a change of orientation for this region. The $\mathrm{SdhB}$ subunit is shaped like a butterfly and organized into two domains. The SdhC and SdhD transmembrane helices exhibit a similar fold and are designated $\mathrm{I}_{\text {to }} \mathrm{VI}^{8}$ (Fig. 2b). The $\mathrm{N}$ terminus of SdhD subunit contains a long loop protruding into the cytoplasm (Fig. 2c), mediating the interactions between adjacent monomers in the assembly. In addition to the four known subunits of complex II, there is extra density corresponding to a transmembrane helix (Supplementary Fig. 3a), mainly located near the SdhC subunit (Fig. 2a, b). The density could be assigned to a previously undescribed subunit in the complex we identify as SdhF. Because of its much lower molecular weight, it was not detected on the SDS-PAGE. However, it could be detected by MS (Supplementary Table 1). We refer to it as SdhF because SdhE has already been used as an assembly factor of the SdhA subunit in $\mathrm{SQR}$ and $\mathrm{QFR}^{30,31}$. It is worth noting that the homologous sequences of SdhF subunit are mainly found in mycobacteria (Supplementary Fig. 5), which suggests that the analogs of Sdh2 complex might be widely distributed in mycobacteria. 
a
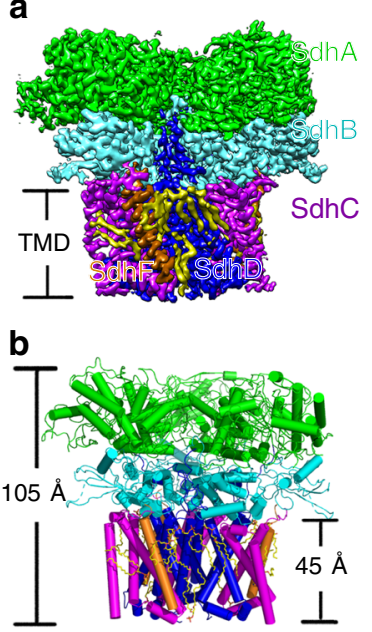
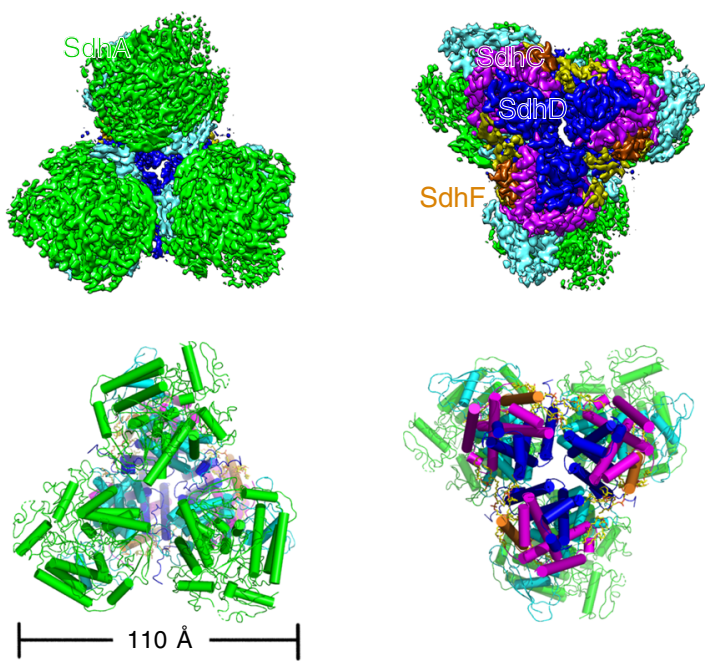

Fig. 1 Overall architecture of the Sdh2 trimer from M. smegmatis. a Cryo-EM map of Sdh2 trimer at $2.8 \AA$ resolution. Left: front view; middle: top view (from cytoplasm); right: bottom view (from periplasm). SdhA, SdhB, SdhC, SdhD, and SdhF subunits are colored in green, cyan, purple, blue, and orange individually. Lipids are colored in yellow. b Cartoon representation of Sdh2 trimer, using the same color scheme as above.

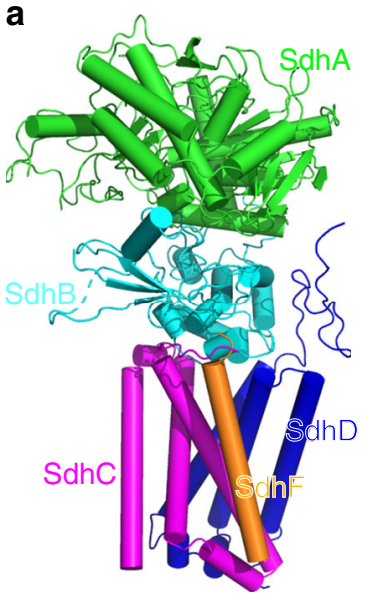

b

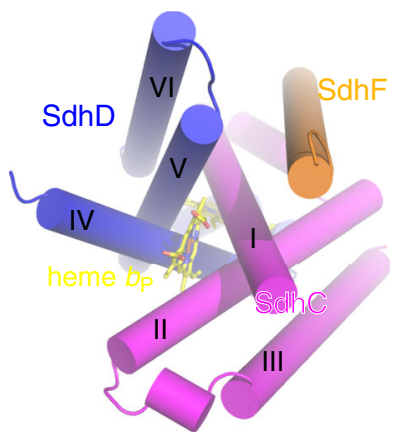

C

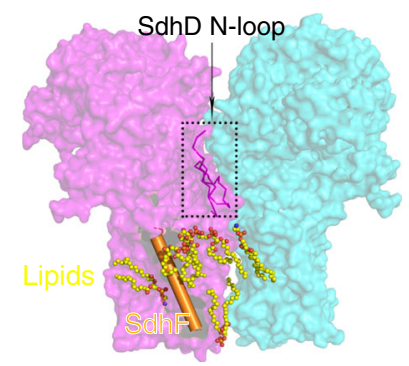

d

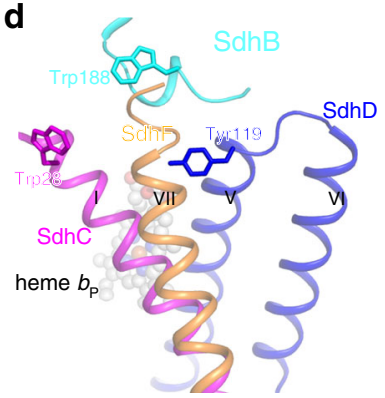

Fig. 2 Location and interaction of SdhF subunit in Sdh2 protein. a One copy of the complex is composed of five subunits SdhA, SdhB, SdhC, SdhD, and $\mathrm{SdhF}$, which are colored green, cyan, purple, blue, and orange, respectively. b Sequential display of transmembrane helices of the SdhC, SdhD, and SdhF subunits. c The distribution of phospholipids (shown in spheres) and SdhF subunit (shown in cylindric cartoon) and SdhD N-loop (shown in ribbons). d SdhF subunit is located in front of the conserved $Q_{p}$-binding pocket.

All the prosthetic groups (FAD, $[2 \mathrm{Fe}-2 \mathrm{~S}],[4 \mathrm{Fe}-4 \mathrm{~S}],[3 \mathrm{Fe}-4 \mathrm{~S}]$, and two $b$-type heme groups) were unambiguously assigned into the cryo-EM map (Supplementary Fig. 3b). It has been previously shown that different residues in the FAD-binding site are responsible for either efficient $\mathrm{SQR}$ or QFR catalytic activity ${ }^{32}$.
Analysis of the amino acids in M. smegmatis Sdh2 (e.g., key residues such as Gln-A48) shows it functions as an $\mathrm{SDH}^{23}$. The three different iron-sulfur clusters of $\mathrm{SdhB}$ subunit are coordinated by conserved cysteine residues observed previously in SQRs and QFRs $s^{6,8,9,11}$. Two $b$-type hemes, denoted as $b_{\mathrm{P}}$ heme and $b_{\mathrm{D}}$ heme based on their proximity to the hydrophilic subunits, are located in the membrane-spanning regions of the subunits SdhC and $\mathrm{SdhD}$, and their axial ligands are His D107-His C87 and His D65-His C47, respectively. Indeed, the bis-histidine axial ligation is conserved throughout all complex IIs, regardless of the number of heme cofactors $6,8,9$.

Fifteen phospholipid molecules and six menaquinone/menaquinols are all clearly observed in the structure (Supplementary Figs. $3 \mathrm{c}$ and 4a), which is in agreement with MS (Supplementary Fig. 1f). However, no density for APM (acylated phosphatidylinositol mannoside) was observed, despite the fact that it was observed by MS. According to the distribution of lipids shown by MS, the proportion of APM was very low in comparison to other lipids in the sample. In the present structure, cardiolipin (CL), phosphatidylethanolamine (PE), phosphatidylinositol (PI), and phosphatidic acid (PA) molecules are mainly distributed in the cleft in the transmembrane space formed by neighboring subunits (Fig. $2 \mathrm{c}$ and Supplementary Fig. 3c), playing roles in mediating the interaction of subunits within protomers of the assembly and interactions between neighboring protomers of the assembly ${ }^{27}$.

SdhF subunit blocks the canonical $Q_{\mathrm{p}}$-binding site. The subunit structure of complex II is highly conserved across bacteria, animals, and fungi, consisting of the four subunits SdhA, B, C, and D. However, other subunits have also been detected ${ }^{30,33}$, which play key roles in regulating the stability, activity, and incorporation of essential cofactors in many multi-subunit protein complexes in biogenesis ${ }^{34}$. Here, we have identified a $3 \mathrm{kDa}$ subunit SdhF, which has only one transmembrane helix for its secondary structure, which is located near the transmembrane helix I of SdhC (Fig. 2a, b). SdhF subunit forms contacts with transmembrane helices I, V and SdhB subunit. There are also extensive interactions with molecules of PA, PE, PI, and CL (Fig. $2 \mathrm{c}$ and Supplementary Fig. 3c).

There are two quinone-binding sites observed in the structures of porcine SQR and E. coli QFR, which have been termed $\mathrm{Q}_{\mathrm{P}}$ and $\mathrm{Q}_{D}$ sites ${ }^{6,8}$, located on opposite sides of the membrane-spanning 

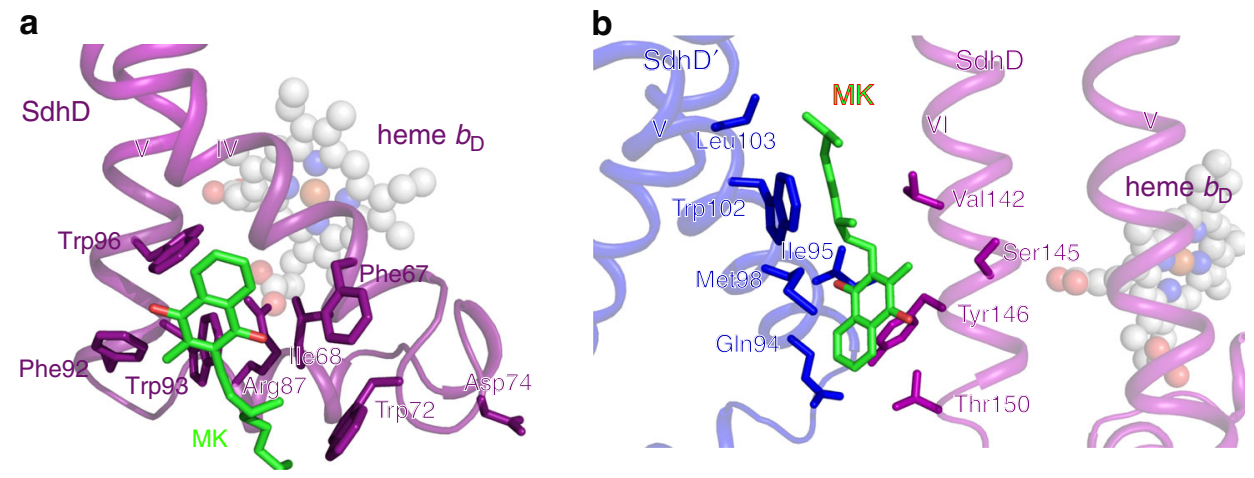

C

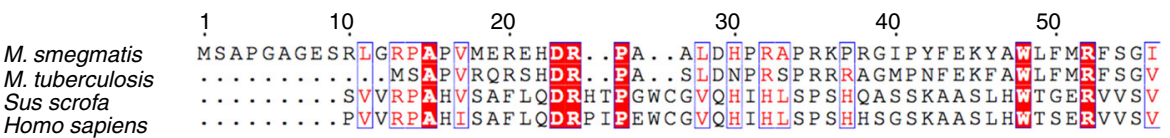

Homo sapiens

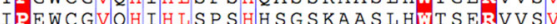

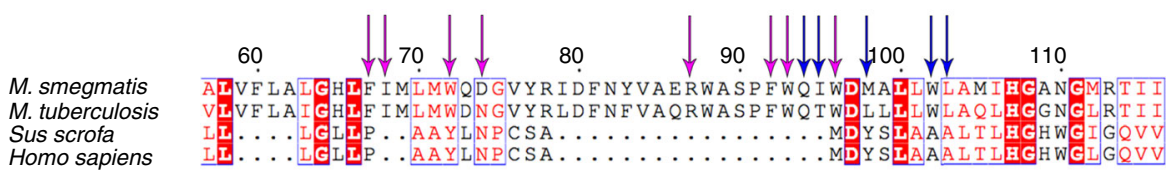

Sus scrofa

Homo sapiens

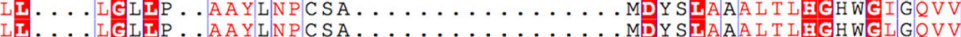

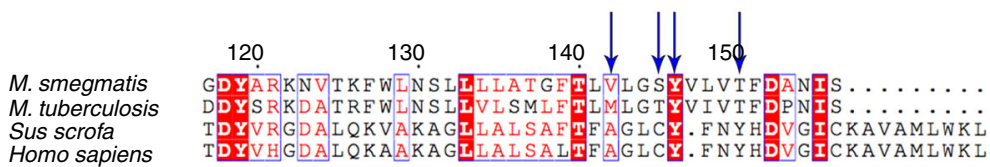

Fig. 3 Quinone binding sites analysis in M. smegmatis Sdh2 trimer. a Close-up views of $Q_{D 1}$ binding site. The heme $b_{\mathrm{P}}$ nearby is shown in spheres. b Close-up views of $\mathrm{Q}_{\mathrm{D} 2}$ located between two neighboring SdhD subunits. c Sequence alignments of the SdhD subunits from M. smegmatis, M. tuberculosis, Sus scrofa, and Homo sapiens. Residues corresponding to $Q_{D 1}$ site and to $Q_{D 2}$ site are labeled with purple and blue arrows, respectively.

region. In terms of the $\mathrm{Q}_{\mathrm{P}}$ site, they are very strongly conserved between mitochondrial SQR and E. coli $\mathrm{SQR}^{6,11}$. This site is also well conserved in our structure (Supplementary Fig. 4b). However, the equivalent $\mathrm{Q}_{\mathrm{P}}$-binding pocket in M. smegmatis Sdh2 structure is completely blocked by the SdhF subunit (Fig. 2d), making this site totally inaccessible from the outside. In addition, we could not find any other density that could be assigned as menaquinone in this binding pocket. Hence, we suggest that the $\mathrm{Q}_{\mathrm{P}}$-binding site does not exist in the present structure, which is consistent with a previous study ${ }^{35}$.

QD-binding site contributes to the structure-based drug discovery. Quinone binding sites in respiratory complexes have varied sequences and specificities depending on the species of origin. They are of great interest, because they are often considered as clinical drug targets ${ }^{27}$. As mentioned above, there is no Qp binding site in M. smegmatis Sdh2. However, a $\mathrm{Q}_{\mathrm{D}}$ binding site has been identified by means of bound inhibitor in porcine $\mathrm{SQR}^{6}$ (Supplementary Fig. 4c), which is termed the $\mathrm{Q}_{\mathrm{D} 1}$ binding site in our structure. In the porcine SQR, Trp-D134, Tyr-D61 (equivalent residues Trp-D88 and Tyr-D83 in our structure) and Lys-D135, Asp-D69 (equivalent residues Arg-D87 and Asp-D74 in present structure) in this pocket are considered to confer specificity for quinone binding and take the functions of quinone protonation, respectively. Coincidentally, we also identified a menaquinone close to the predicted $\mathrm{Q}_{\mathrm{D}}$-binding pocket considered analogous to that in porcine SQR (Fig. 3a). It is surrounded by the side chains of residues Phe-D67, Ile-D68, Trp-D72, Phe-D92, Trp-D93, and Trp-D96. The edge-to-edge distance from $\mathrm{MK}$ to $b_{\mathrm{D}}$ heme is $15 \AA$, exceeding the $14 \AA$ limit for physiological electron transfer ${ }^{36}$, and there are no observed hydrogen bonds to the carbonyl groups of $\mathrm{MK}$, which are needed to couple proton abstraction and the accepting of electrons. Besides that, a salt bridge is observed between Arg-D87 and the ring C-propionate of $b_{\mathrm{D}}$ heme, indicating that the $b_{\mathrm{D}}$ heme is in oxidative status in the present structure ${ }^{37}$. Thus, we speculate that the endogenous menaquinone, close to $\mathrm{Q}_{\mathrm{D} 1}$-binding pocket, might be a representation of the reduced product $\mathrm{MKH}_{2}$, which is leaving the $\mathrm{Q}_{\mathrm{D} 1}$ site.

We also assigned a second bound $\mathrm{MK} / \mathrm{MKH}_{2}$ to the cryo-EM density map adjacent to the $b_{\mathrm{D}}$ heme. Henceforth, we refer to this as the $\mathrm{Q}_{\mathrm{D} 2}$ site (Supplementary Fig. 4a). This binding site is located within the interface formed by membrane-spanning region of adjacent protomers in the assembly. This is also suggested in other studies, which showed that there is a quinone-binding site located between adjacent monomers in the trimeric Sdh complex from $T$. thermophiles ${ }^{20,21}$. The bound $\mathrm{MK} / \mathrm{MKH}_{2}$ interacts with the side chains of Gln-D94, Ile-D95, Met-D98, Trp-D102, and Leu-D103 of transmembrane helix $\mathrm{V}$ in one protomer, and the Val-D142, SerD145, Tyr-D146, Val-D149, and Thr-D150 of transmembrane helix VI in an adjacent protomer of the assembly (Fig. 3b). As mentioned above, based on the relationship of proton abstraction and accepting electrons, MK should bind deeper close to polar residues, such as Gln-D94, Tyr-D146, and Thr-D150. Interestingly, the edge-to-edge distances between $\mathrm{MK} / \mathrm{MKH}_{2}$ and the neighboring $b_{\mathrm{D}}$ hemes are 9 and $11 \AA$, respectively. These findings suggest that the $\mathrm{Q}_{\mathrm{D} 2}$ site can receive the electrons from one or two of the $b_{\mathrm{D}}$ hemes in adjacent protomers, and the bound molecule in the present structure is a representation of fully reduced MK. According to the kinetic characterization of the $\mathrm{Q}_{\mathrm{D} 1 / 2}$ mutants of 
Sdh2 using menadione/DCIP (succinate-2,6-dichlorophenolindophenol) assay (Supplementary Fig. 6), the $k_{\text {cat }}$ values of the $\mathrm{Q}_{\mathrm{D} 1}$ and $\mathrm{Q}_{\mathrm{D} 2}$ sites are $2.16 \pm 0.04 \mathrm{~s}^{-1}$ and $1.48 \pm 0.04 \mathrm{~s}^{-1}$, respectively, which are all lower than that of wild-type Sdh2 enzyme. The mutant $\mathrm{Q}_{\mathrm{D} 2}$ more severely affects the catalytic activity in comparison to that of mutant $\mathrm{Q}_{\mathrm{D} 1}$. Their $K_{\mathrm{m}}$ values are $72.67 \pm$ 5.73 and $53.54 \pm 6.36 \mu \mathrm{M}$, respectively, approaching that of the wild-type Sdh2 enzyme. These data confirm that the identified $\mathrm{Q}_{\mathrm{D} 1 / 2}$ sites are the substrate-binding sites.

Complex II enzymes have been largely neglected for drug development in bacterial pathogens, despite them playing an essential role in bacterial metabolism and respiration ${ }^{19}$. Mammalian complex II is coupled to ubiquinone, whereas mycobacterial enzymes use menaquinone, providing opportunities for developing selective inhibitors. It has been reported that siccanin, a ubiquinone analog, can selectively inhibit the Sdhs from different species ${ }^{38}$, raising the possibility that menaquinone-based drugs can be specific against Sdhs and therefore be safe for use in humans. As for the $\mathrm{Q}_{\mathrm{D} 1}$ site (Fig. 3a), sequence alignments indicate the residues of quinone-binding site of $\mathrm{Sdh} 2$ protein from $M$. tuberculosis and $M$. smegmatis are highly similar, suggesting that they have a similar quinone-binding mechanism. However, despite the similarity of residues of the $\mathrm{Q}_{\mathrm{D} 1}$ sites between porcine and human, they are different from that of mycobacteria (Fig. 3c). Moreover, the $\mathrm{Q}_{\mathrm{D} 1}$-binding pocket in porcine $\mathrm{SQR}$ is formed by transmembrane helices IV, $\mathrm{V}$ and the $\mathrm{N}$-terminal $\alpha$-helix that stretches from the $\mathrm{N}$ terminus of transmembrane helix VI, but the binding pocket of $\mathrm{Q}_{\mathrm{D} 1}$ site $M$. smegmatis Sdh2 is involved in transmembrane helices IV, V and one bridging $\alpha$-helix of them. This significant difference can be observed based on the superposition of their structures (Supplementary Fig. 4c). In terms of the $\mathrm{Q}_{\mathrm{D} 2}$ site (Fig. 3b), it is formed by the neighboring protomers, but the mammalian $\mathrm{SQR}$ exists only in monomeric form ${ }^{6}$ (Supplementary Fig. 4b). These observations thus suggest that the $\mathrm{Q}_{\mathrm{D}}$ sites in $M$. tuberculosis are selective compared with those in the human counterpart. Thus, it is reasonable to suggest that this is a good antimycobacterial drug target, which is species selective.

Electron transfer in the trimer offers clues to the cooperativity. In the M. smegmatis Sdh2 structure, the succinate oxidation site and $\mathrm{MK}$ reduction site are connected by a chain of redox centers including $\mathrm{FAD},[2 \mathrm{Fe}-2 \mathrm{~S}],[4 \mathrm{Fe}-4 \mathrm{~S}],[3 \mathrm{Fe}-4 \mathrm{~S}], b_{\mathrm{P}}$ heme and $b_{\mathrm{D}}$ heme (Fig. 4a). Bacillus species SQRs also use diheme-containing $\mathrm{SQRs}{ }^{18}$, and their $b_{\mathrm{D}}$ hemes are essential for electron transfer to menaquinone ${ }^{35}$. The EPR data (Supplementary Fig. 1e) also show that the electrons pass through $b_{\mathrm{D}}$ heme during menaquinone reduction. The distances between adjacent redox centers allow for efficient electron transfer ${ }^{36}$.

Previous studies have demonstrated that the monomeric and trimeric structure of the diheme-containing SQRs from $T$. thermophiles have SDH activities, but only the trimeric form exhibits positive cooperativity under high temperature ${ }^{20,21}$. The authors proposed that each monomer contains an MK binding site and can cooperatively interact with other MK-binding sites also exist within the trimeric structure. As described above, we have confirmed two substrate-binding sites (sites $\mathrm{Q}_{\mathrm{D} 1}$ and $\mathrm{Q}_{\mathrm{D} 2}$ ) by analyzing the catalytic activity of the mutant and wild-type Sdh2, and the features of Sdh2 structure. So, the structure here can provide a framework for understanding such a cooperative process. In addition, these data also demonstrate that the oxidized menaquinones bound to the $\mathrm{Q}_{\mathrm{D} 1 / 2}$ sites can accept the electrons from the $b_{\mathrm{D}}$ heme. But whether the menaquinone bound to the $\mathrm{Q}_{\mathrm{D} 2}$ site accesses to the electrons from the $b_{\mathrm{D}}$ hemes of adjacent monomers at the same time is unclear. It is worth noting that
Sdh2 plays essential roles under hypoxia in $M$. smegmatis $^{23}$ and M. tuberculosis ${ }^{24}$, suggesting that its trimeric nature may work cooperatively to meet the energy demand for survival or to effectively regulate energy metabolism under hypoxia ${ }^{26}$.

Therefore, the electrons are transferred from the succinate to menaquinone bound to $\mathrm{Q}_{\mathrm{D} 1}$ and $\mathrm{Q}_{\mathrm{D} 2}$ sites, achieving the oxidation of succinate and reduction of menaquinone (Fig. $4 \mathrm{~b}$ ). In Bacillus subtilis ${ }^{17}$ and Bacillus lichenformis ${ }^{18}$, it has been proposed that this type of reverse electron transport is driven by the proton potential, providing the driving force for MK reduction. As an analog of SQR, W. succinogenes QFR has corresponding pathways to proton transfer, in which Glu-C60 and Glu-C180 play crucial functional roles ${ }^{14,15,39}$. Although the present structure lacks an acidic residue at the equivalent position of Glu-C180, there is a well-conserved acidic residue Glu-D86 close to the $\mathrm{Q}_{\mathrm{D} 1}$ site, corresponding to Glu-C66 in $W$. succinogenes $\mathrm{QFR}^{35}$. This finding suggests that the Glu-D86 pathway participates in the proton transfer among the dihemecontaining SQRs, and there is no canonical E-pathway-producing proton potential across the membrane. However, it has been proposed that one proton can be transferred directly from the quinol oxidation site to the Glu-C180 pathway in dihemecontaining QFRs with the help of the ring C-propionate of $b_{\mathrm{D}}$ heme ${ }^{40}$, indicating the existence of a proton transfer pathway between the $\mathrm{Q}_{\mathrm{D} 2}$ site and ring C-propionate of $b_{\mathrm{D}}$ heme. Interestingly, the $\mathrm{Q}_{\mathrm{D} 2}$ site is located near the ring C-propionate of $b_{\mathrm{D}}$ heme of neighboring protomers in this structure, which suggests that during $M K$ reduction at the $\mathrm{Q}_{\mathrm{D} 2}$ site protons may be uptaken through this pathway. Consequently, the Glu-D86 site is considered as the proton entry site, driving the reduction of $\mathrm{MK}$ molecules bound to $\mathrm{Q}_{\mathrm{D} 1}$ and $\mathrm{Q}_{\mathrm{D} 2}$ sites (Fig. 4b). Certainly, we cannot rule out the possibility of unidentified proton transfer pathways.

We have determined the cryo-EM structure of a trimeric Sdh from M. smegmatis at $2.8 \AA$, which identifies a non-covalently bound membrane-anchored subunit referred to as $\mathrm{SdhF}$ and shows that the electron transfer pathway is cooperative. Our study further provides a framework for rational structure-based anti-tuberculosis drug discovery.

\section{Methods}

Bacteria strain and culture. By means of homologous recombination, the Sdh2D gene (MSMEG_1671) was cloned into the expression plasmid pVV16 with a 10× His tag at the $\bar{C}$ terminus. Mycobacterium smegmatis mutant strain, $\mathrm{mc}^{2} 51^{41}$, was chosen for expression of the protein. The primers are listed in the Supplementary Table 4 . Reconstructed cell solutions were cultured until the $\mathrm{OD}_{600}$ reached $\sim 1.5$ and then harvested by centrifugation for $30 \mathrm{~min}$ at 4000 r.p.m.

Protein purification and characterization. Cells including the interest protein were lysed through a high-pressure cell disrupter at $4{ }^{\circ} \mathrm{C}$ and 1200 bar. The lysate was centrifuged at 14,000 r.p.m. for $10 \mathrm{~min}$ to remove cell debris and non-lysed cells. The resulting supernatant was centrifuged at 36,900 r.p.m. for $1 \mathrm{~h}$ in a Ti45 rotor (Beckman) and the membrane pellets were harvested. Membranes of the cells were extracted in buffer (20 mM MOPS, $\mathrm{pH} 7.4,100 \mathrm{mM} \mathrm{NaCl})$, and then stirred slowly at $4{ }^{\circ} \mathrm{C}$ for $3 \mathrm{~h}$ with the $1 \%(\mathrm{w} / \mathrm{v})$ digitonin added. The supernatant after centrifugation was loaded onto a Ni-NTA column in the buffer containing $20 \mathrm{mM}$ MOPS, $\mathrm{pH} 7.4,100 \mathrm{mM} \mathrm{NaCl}$, and $0.1 \%(\mathrm{w} / \mathrm{v})$ digitonin, and the eluted fraction including the protein of interest was loaded onto a Superdex 200 (GE Healthcare) column equilibrated in a buffer containing $20 \mathrm{mM}$ MOPS, $\mathrm{pH} 7.4,100 \mathrm{mM} \mathrm{NaC}$ and $0.1 \%(\mathrm{w} / \mathrm{v})$ digitonin. The peak fractions were pooled and concentrated to $5 \mathrm{mg} / \mathrm{mL}$.

To detect the protein components, the concentrated protein sample and the strips after SDS-PAGE analysis were subjected to MS analysis at the National Facility for Protein Science in Shanghai (NFPS). The protein sample was analyzed by BN-PAGE with NTB staining ${ }^{42}$. In order to use normal-phase liquid chromatography-MS (LCMS) to detect phospholipids and free mycolic acids ${ }^{43,44}$ we processed the samples as described previously study ${ }^{27}$. In addition, the levels of MK species were also quantified using a reverse-phase LCMS method ${ }^{45}$.

EPR spectra were acquired as previously described using a Bruker X-band (9.4 GHz) EMX plus 10/12 spectrometer with ESR-910 flowing helium cryostat, at a temperature of $15 \mathrm{~K}$, and using a 5-Gauss modulation amplitude at $100 \mathrm{kHz}$ 
a
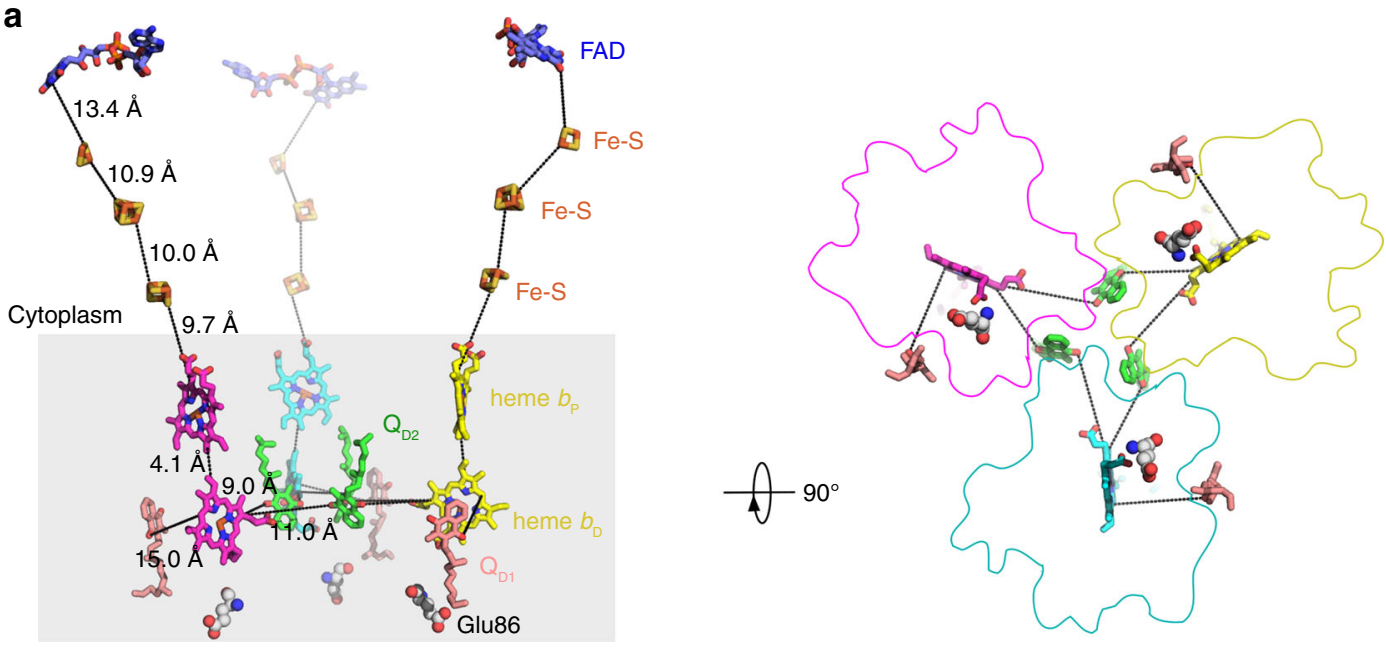

Periplasm

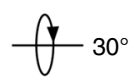

\section{b}
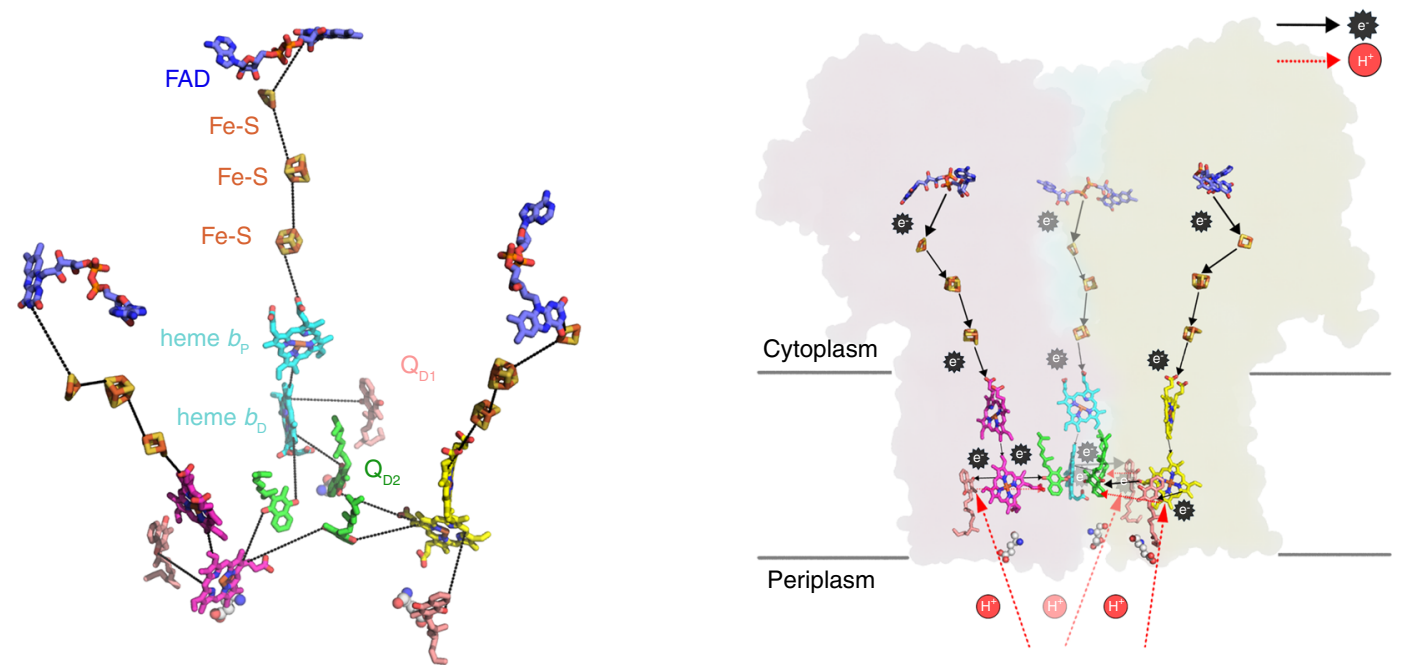

Fig. 4 Electron/proton transfer pathway in the Sdh2 trimer. a The prosthetic groups, FAD, Fe-S clusters, hemes, and MK, are shown in stick or sphere. The edge-to-edge distances between adjacent prosthetic groups are labeled in black dashed lines. b Representation of electron and proton transfer way between adjacent protomers in the trimer. Electron transfer directions are shown in black arrows. Proton transfer pathways are shown by the red arrows.

under non-saturating MW power conditions $(200 \mu \mathrm{W})^{27}$. To reveal different electron transfer components in the complex, samples in the "air-oxidized" state (as isolated) or "reduced" state (reduced by addition of $50 \mu \mathrm{M}$ succinate or further reduced by $200 \mu \mathrm{M}$ dithionite) were prepared $20,46-50$. The addition of reducing agents was done at room temperature followed by vortexing and freezing of the sample in liquid nitrogen within $5 \mathrm{~min}$ of addition.

Characterization of enzyme activity. The protein samples were obtained by using plasmid pVV16 that is used for expression in Mycobacterium smegmatis, and the primers used in this study are listed in the Supplementary Table 4. The SQR activity of two different mutants $\mathrm{Q}_{\mathrm{D} 1 / 2}$ and wild-type of Sdh2 was determined using the DCIP assay according to a previous study ${ }^{23}$. In the present study, the menadione was selected as the intermediate electron acceptor. The reaction mixture contained $20 \mathrm{mM}$ MOPS (pH 7.4), 0.005\% LMNG (lauryl maltose neopentyl glycol), $0.025-1.6 \mathrm{mM}$ sodium succinate, $1 \mathrm{mM}$ menadione, and $100 \mu \mathrm{M}$ DCIP. The reaction was initiated by the addition of DCIP into the mixture. All assays were performed at $37^{\circ} \mathrm{C}$, using a Molecular Devices iD3 Reader. Data were processed with GraphPad Prism 6.

Cryo sample acquisition and data collection. Three microliters of aliquots of digitonin-solubilized protein at a concentration of $5 \mathrm{mg} / \mathrm{mL}$ were applied to $\mathrm{H}_{2} / \mathrm{O}_{2}$ glow-discharged 300-mesh Quantifoil R1.2/1.3 grids (Quantifoil, Micro Tools
$\mathrm{GmbH}$, Germany). Solution-absorbed grids were blotted for $2.5 \mathrm{~s}$ with force 1 at $8^{\circ} \mathrm{C}$ and $100 \%$ humidity, and then plunged into pre-cooled liquid ethane using an FEI Vitrobot. Cryo-EM images were collected on a Titan Krios $300 \mathrm{kV}$ electron microscope (Thermo Company), equipped with K3 Summit direct electron detector camera (Gatan). Images were recorded at $\times 29,000$ magnification with a calibrated super-resolution pixel size $0.82 \AA$ /pixel. The exposure time was set to $2 \mathrm{~s}$ with a total accumulated dose of 50 electrons per $\AA^{2}$. All images were automatically recorded using SerialEM ${ }^{51}$ with a defocus range from 1.2 to $2.2 \mu \mathrm{m}$. A total of 5420 images were collected.

Image processing. All dose-fractioned images were motion-corrected and doseweighted by the MotionCorr 2 software ${ }^{52}$ and their contrast transfer functions were estimated by $\mathrm{Gctf}^{53}$. A total of 1,132,663 particles were picked automatically and extracted with a box size of 384 pixels in cryoSPARC ${ }^{54}$. The following $2 \mathrm{D}, 3 \mathrm{D}$ classifications and refinements were all performed in cryoSPARC ${ }^{54}$. A total of 564,244 particles were selected after two rounds of 2D classification. Hundred thousand particles were used to do $\mathrm{Ab}$ initio reconstruction in four classes, and then these four classes were used as $3 \mathrm{D}$ volume templates for heterogeneous refinement with all selected particles. In all, 461,385 particles converged into one class, yielding a $4.7 \AA$ initial map. Then, this particle set was used to do homogeneous refinement, yielding $2.9 \AA$. After non-uniform refinement, the final resolution was $2.8 \AA$. 
Model building and refinement. An atomic model of Sdh2 was manually built in Coot $0.8 .9 .1^{55}$ using the crystal structure of porcine Sdh (PDB code: $\left.1 \mathrm{ZOY}\right)^{6}$ as a template. From the local resolution map, the transmembrane domains had a relative high resolution, while the water-soluble subunits are at lower resolution in some regions. Thus, we were able to model correctly the side chains of the transmembrane subunits and the majority of water-soluble subunits. From the map and MS results, we were able to identify the relevant redox centers, phospholipids, MKs, and a subunit SdhF. All prosthetic groups and phospholipids models were generated using the elbow module in PHENIX with simple constraints ${ }^{56}$. These small molecules were docked into the map and refined in Coot 0.8.9.1. The differentiation of phospholipids is based on a previous study ${ }^{27}$. Final maps were sharpened automatically by applying a $B$-factor of $93.5 \AA^{2}$ using cryoSPARC and subjected to real-space refinement using PHENIX. The local resolution map was calculated with ResMap ${ }^{57}$. All reported resolutions were based on the goldstandard FSC 0.143 criteria $^{58}$. FSCwork and FSCtest were conducted to check for overfitting 59

All the figures were created by means of UCSF Chimera ${ }^{60}$ or PyMOL ${ }^{61}$.

Reporting summary. Further information on research design is available in the Nature Research Reporting Summary linked to this article.

\section{Data availability}

The accession number for the 3D cryo-EM density map reported in present study is EMD-0981. The accession number for the coordinates for the structure reported in this study is 6LUM. Source data are provided with this paper.

Received: 16 January 2020; Accepted: 24 July 2020;

Published online: 25 August 2020

\section{References}

1. Mitchell, P. Coupling of phosphorylation to electron and hydrogen transfer by a chemi-osmotic type of mechanism. Nature 191, 144-148 (1961).

2. Saraste, M. Oxidative phosphorylation at the fin de siecle. Science 283, 1488-1493 (1999).

3. Iverson, T. Catalytic mechanisms of complex II enzymes: a structural perspective. Biochem. Biophys. Acta 1827, 648-657 (2013).

4. Hirsch, C. A., Rasminsky, M., Davis, B. D. \& Lin, E. C. A fumarate reductase in Escherichia coli distinct from succinate dehydrogenase. J. Biol. Chem. 238, 3770-3774 (1963).

5. Hards, K., Rodriguez, S. M., Cairns, C. \& Cook, G. M. Alternate quinone coupling in a new class of succinate dehydrogenase may potentiate mycobacterial respiratory control. FEBS Lett. 593, 475-486 (2019).

6. Sun, F. et al. Crystal structure of mitochondrial respiratory membrane protein complex II. Cell 121, 1043-1057 (2005).

7. Huang, L.-S. et al. 3-Nitropropionic acid is a suicide inhibitor of mitochondrial respiration that, upon oxidation by complex II, forms a covalent adduct with a catalytic base arginine in the active site of the enzyme. J. Biol. Chem. 281, 5965-5972 (2006).

8. Iverson, T. M., Luna-Chavez, C., Cecchini, G. \& Rees, D. C. Structure of the Escherichia coli fumarate reductase respiratory complex. Science 284, 1961-1966 (1999).

9. Lancaster, C. R. D., Kröger, A., Auer, M. \& Michel, H. Structure of fumarate reductase from Wolinella succinogenes at $2.2 \AA$ resolution. Nature 402, 377 (1999).

10. Guan, H.-H. et al. Structural insights into the electron/proton transfer pathways in the quinol: fumarate reductase from Desulfovibrio gigas. Sci. Rep. 8, 14935 (2018).

11. Yankovskaya, V. et al. Architecture of succinate dehydrogenase and reactive oxygen species generation. Science 299, 700-704 (2003).

12. Lancaster, C. R. D. The di-heme family of respiratory complex II enzymes. Biochem. Biophys. Acta 1827, 679-687 (2013).

13. Lancaster, C. R. D., Betz, Y. M., Heit, S. \& Lafontaine, M. A. Transmembrane electron and proton transfer in diheme-containing succinate: quinone oxidoreductases. Isr. J. Chem. 57, 370-380 (2017).

14. Lancaster, C. R. D. et al. Experimental support for the "E pathway hypothesis" of coupled transmembrane $\mathrm{e}^{-}$and $\mathrm{H}+$ transfer in dihemic quinol: fumarate reductase. Proc. Natl Acad. Sci. USA 102, 18860-18865 (2005).

15. Madej, M. G., Nasiri, H. R., Hilgendorff, N. S., Schwalbe, H. \& Lancaster, C. R. D. Evidence for transmembrane proton transfer in a dihaem-containing membrane protein complex. EMBO J. 25, 4963-4970 (2006).

16. Thauer, R. K., Jungermann, K. \& Decker, K. Energy conservation in chemotrophic anaerobic bacteria. Bacteriol. Rev. 41, 100 (1977).

17. Schirawski, J. \& Unden, G. Menaquinone-dependent succinate dehydrogenase of bacteria catalyzes reversed electron transport driven by the proton potential. Eur. J. Biochem. 257, 210-215 (1998).
18. Madej, M. G. et al. Experimental evidence for proton motive force-dependen catalysis by the diheme-containing succinate: menaquinone oxidoreductase from the Gram-positive bacterium Bacillus licheniformis. Biochemistry 45, 15049-15055 (2006).

19. Hards, K. et al. Two for the price of one: attacking the energetic-metabolic hub of mycobacteria to produce new chemotherapeutic agents. Prog. Biophys. Mol. Biol. IF 2.175 (2019).

20. Kolaj-Robin, O. et al. Biochemical and biophysical characterization of succinate: quinone reductase from Thermus thermophilus. Biochem. Biophys. Acta 1807, 68-79 (2011).

21. Kolaj-Robin, O., Noor, M. R., O'Kane, S. R., Baymann, F. \& Soulimane, T. Atypical features of Thermus thermophilus succinate: quinone reductase. PLoS ONE 8, e53559 (2013).

22. Kurokawa, T. \& Sakamoto, J. Purification and characterization of succinate: menaquinone oxidoreductase from Corynebacterium glutamicum. Arch. Microbiol. 183, 317-324 (2005).

23. Pecsi, I. et al. Essentiality of succinate dehydrogenase in Mycobacterium smegmatis and its role in the generation of the membrane potential under hypoxia. MBio 5, e01093-01014 (2014).

24. Hartman, T. et al. Succinate dehydrogenase is the regulator of respiration in Mycobacterium tuberculosis. PLoS Pathog. 10, e1004510 (2014).

25. Baek, S.-H., Li, A. H. \& Sassetti, C. M. Metabolic regulation of mycobacterial growth and antibiotic sensitivity. PLoS Biol. 9, e1001065 (2011).

26. Berney, M. \& Cook, G. M. Unique flexibility in energy metabolism allows mycobacteria to combat starvation and hypoxia. PLoS ONE 5, e8614 (2010).

27. Gong, H. et al. An electron transfer path connects subunits of a mycobacterial respiratory supercomplex. Science 362, eaat8923 (2018).

28. Tomasiak, T. M., Maklashina, E., Cecchini, G. \& Iverson, T. M. A threonine on the active site loop controls transition state formation in Escherichia coli respiratory complex II. J. Biol. Chem. 283, 15460-15468 (2008).

29. Starbird, C. et al. New crystal forms of the integral membrane Escherichia coli quinol: fumarate reductase suggest that ligands control domain movement. $J$. Struct. Biol. 202, 100-104 (2018).

30. Maher, M. J., Herath, A. S., Udagedara, S. R., Dougan, D. A. \& Truscott, K. N. Crystal structure of bacterial succinate: quinone oxidoreductase flavoprotein SdhA in complex with its assembly factor SdhE. Proc. Natl Acad. Sci. USA 115 , 2982-2987 (2018).

31. Sharma, P., Maklashina, E., Cecchini, G. \& Iverson, T. Crystal structure of an assembly intermediate of respiratory complex II. Nat. Commun. 9, 274 (2018).

32. Maklashina, E. et al. Fumarate reductase and succinate oxidase activity of Escherichia coli complex II homologs are perturbed differently by mutation of the flavin binding domain. J. Biol. Chem. 281, 11357-11365 (2006).

33. Schikowsky, C., Senkler, J. \& Braun, H. P. SDH6 and SDH7 contribute to anchoring succinate dehydrogenase to the inner mitochondrial membrane in Arabidopsis thaliana. Plant Physiol. 173, 1094-1108 (2017).

34. Maghool, S. et al. Structural and functional characterization of the mitochondrial complex IV assembly factor Coa6. Life Sci. Alliance 2 e201900458 (2019).

35. Matsson, M., Tolstoy, D., Aasa, R. \& Hederstedt, L. The distal heme center in Bacillus subtilis succinate: quinone reductase is crucial for electron transfer to menaquinone. Biochemistry 39, 8617-8624 (2000).

36. Page, C. C., Moser, C. C., Chen, X. \& Dutton, P. L. Natural engineering principles of electron tunnelling in biological oxidation-reduction. Nature 402, 47 (1999).

37. Haas, A. H. \& Lancaster, C. R. D. Calculated coupling of transmembrane electron and proton transfer in dihemic quinol: fumarate reductase. Biophys. J. 87, 4298-4315 (2004).

38. Mogi, T. et al. Siccanin rediscovered as a species-selective succinate dehydrogenase inhibitor. J. Biochem. 146, 383-387 (2009).

39. Lancaster, C. R. D. et al. Essential role of Glu-C66 for menaquinol oxidation indicates transmembrane electrochemical potential generation by Wolinella succinogenes fumarate reductase. Proc. Natl Acad. Sci. USA 97, 13051-13056 (2000).

40. Madej, M. G., Müller, F. G., Ploch, J. \& Lancaster, C. R. D. Limited reversibility of transmembrane proton transfer assisting transmembrane electron transfer in a dihaem-containing succinate: quinone oxidoreductase. Biochem. Biophys. Acta 1787, 593-600 (2009).

41. Li, X., Liu, F., Hu, Y. \& Mi, K. Draft genome sequence of mc251, a highly hydrogen peroxide-resistant Mycobacterium smegmatis mutant strain. Genome Announc. 2, e00092-00014 (2014).

42. Wittig, I., Karas, M. \& Schägger, H. High resolution clear native electrophoresis for in-gel functional assays and fluorescence studies of membrane protein complexes. Mol. Cell. Proteom. 6, 1215-1225 (2007).

43. Lam, S. M. et al. Extensive characterization of human tear fluid collected using different techniques unravels the presence of novel lipid amphiphiles. J. Lipid Res. 55, 289-298 (2014)

44. Shui, G. et al. Mycolic acids as diagnostic markers for tuberculosis case detection in humans and drug efficacy in mice. EMBO Mol. Med. 4, 27-37 (2012). 
45. Lim, L. et al. Lanosterol induces mitochondrial uncoupling and protects dopaminergic neurons from cell death in a model for Parkinson's disease. Cell Death Differ. 19, 416 (2012).

46. Berry, E. A. \& Walker, F. A. Bis-histidine-coordinated hemes in four-helix bundles: how the geometry of the bundle controls the axial imidazole plane orientations in transmembrane cytochromes of mitochondrial complexes II and III and related proteins. J. Biol. Inorg. Chem. 13, 481 (2008).

47. Magalon, A. et al. Heme axial ligation by the highly conserved his residues in helix II of cytochrome $b$ (NarI) of Escherichia coli nitrate reductase A (NarGHI). J. Biol. Chem. 272, 25652-25658 (1997).

48. Pearce, L. L. et al. The resistance of electron-transport chain Fe-S clusters to oxidative damage during the reaction of peroxynitrite with mitochondrial complex II and rat-heart pericardium. Nitric Oxide 20, 135-142 (2009).

49. Tran, Q. M., Rothery, R. A., Maklashina, E., Cecchini, G. \& Weiner, J. H. The quinone binding site in Escherichia coli succinate dehydrogenase is required for electron transfer to the heme b. J. Biol. Chem. 281, 32310-32317 (2006).

50. Beinert, H., Ackrell, B. A., Kearney, E. B. \& Singer, T. P. Iron-sulfur components of succinate dehydrogenase: stoichiometry and kinetic behavior in activated preparations. Eur. J. Biochem. 54, 185-194 (1975).

51. Mastronarde, D. N. Automated electron microscope tomography using robust prediction of specimen movements. J. Struct. Biol. 152, 36-51 (2005).

52. Zheng, S. Q. et al. MotionCor2: anisotropic correction of beam-induced motion for improved cryo-electron microscopy. Nat. Methods 14, 331 (2017).

53. Zhang, K. Gctf: real-time CTF determination and correction. J. Struct. Biol. 193, 1-12 (2016).

54. Punjani, A., Rubinstein, J. L., Fleet, D. J. \& Brubaker, M. A. cryoSPARC: algorithms for rapid unsupervised cryo-EM structure determination. Nat. Methods 14, 290 (2017).

55. Emsley, P., Lohkamp, B., Scott, W. G. \& Cowtan, K. Features and development of Coot. Acta Crystallogr. D 66, 486-501 (2010).

56. Adams, P. D. et al. Advances, interactions, and future developments in the CNS, Phenix, and Rosetta structural biology software systems. Annu. Rev. Biophys. 42, 265-287 (2013)

57. Kucukelbir, A., Sigworth, F. J. \& Tagare, H. D. Quantifying the local resolution of cryo-EM density maps. Nat. Methods 11, 63 (2014)

58. Rosenthal, P. B. \& Henderson, R. Optimal determination of particle orientation, absolute hand, and contrast loss in single-particle electron cryomicroscopy. J. Mol. Biol. 333, 721-745 (2003).

59. Brown, A. et al. Tools for macromolecular model building and refinement into electron cryo-microscopy reconstructions. Acta Crystallogr. D 71, 136-153 (2015).

60. Pettersen, E. F. et al. UCSF Chimera-a visualization system for exploratory research and analysis. J. Comput. Chem. 25, 1605-1612 (2004).

61. Schrodinger, LLC. The PyMOL Molecular Graphics System. Version 1.0 (Schrodinger, LLC, 2010)

\section{Acknowledgements}

We thank Dr. Chao Peng of the Mass Spectrometry System at the National Facility for Protein Science in Shanghai (NFPS), Zhangjiang Lab, SARI, China for data collection and analysis and Prof. Kaixia Mi (CAS Key Laboratory of Pathogenic Microbiology and Immunology, Institute of Microbiology, CAS) for sharing the strain M. smegmatis $\mathrm{mc}^{2}$ 51. We would like to thank the Bio-Electron Microscopy Facility of ShanghaiTech
University, and we would be grateful to Dr. Qianqian Sun for her help of cryo-EM technical support. This work was supported by Grants from the National Key Research and Development Program of China (Grant No. 2017YFC0840300), the Strategic Priority Research Program of the Chinese Academy of Sciences (Grant Nos. XDB37030201, XDB37020203), and the National Natural Science Foundation of China (Grant Nos. 81520108019, 813300237).

\section{Author contributions}

Z.R. conceived and supervised the study. Y.X., X.Z., and H.G. purified the M. smegmatis Sdh2 complex. H.G., X.Z., and Y.X. characterized the spectroscopic features and contents of the samples; X.Z. conducted the enzyme characterization. L.Y. and C.T. performed the EPR experiments and data analysis; S.M.L. and G.S. performed the lipid identification and data analysis. Y.G. collected and processed cryo-EM data and built the structure model; H.G., Q.W., Y.G., X.Z., Y.X., W.W., Y.T., S.Z., Y.Z., W.J., L.W.G., L.-L.W., and Z.R. analyzed the structure and discussed the results and the manuscript was written by H.G., L.G., L.-L.W., Q.W., and Z.R

\section{Competing interests}

L.-L.W. is a founder and consultant of Oxford Biotrans. The other authors declare no competing interests.

\section{Additional information}

Supplementary information is available for this paper at https://doi.org/10.1038/s41467020-18011-9.

Correspondence and requests for materials should be addressed to H.G., Q.W. or Z.R

Peer review information Nature Communications thanks Gregory Cook, Tim Rasmussen and the other, anonymous, reviewer(s) for their contribution to the peer review of this work.

Reprints and permission information is available at http://www.nature.com/reprints

Publisher's note Springer Nature remains neutral with regard to jurisdictional claims in published maps and institutional affiliations.

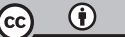

Open Access This article is licensed under a Creative Commons Attribution 4.0 International License, which permits use, sharing, adaptation, distribution and reproduction in any medium or format, as long as you give appropriate credit to the original author(s) and the source, provide a link to the Creative Commons license, and indicate if changes were made. The images or other third party material in this article are included in the article's Creative Commons license, unless indicated otherwise in a credit line to the material. If material is not included in the article's Creative Commons license and your intended use is not permitted by statutory regulation or exceeds the permitted use, you will need to obtain permission directly from the copyright holder. To view a copy of this license, visit http://creativecommons.org/ licenses/by/4.0\%

(C) The Author(s) 2020, corrected publication 2021 